\title{
The Boundary Proportion Differential Control Method of Micro-Deformable Manipulator with Compensator Based on Partial Differential Equation Dynamic Model
}

\author{
Xiangli Pei, Ying Tian *, Minglu Zhang and Ruizhuo Shi \\ School of Mechanical Engineering, Hebei University of Technology, Tianjin 300000, China; \\ pxl_hebut@163.com (X.P.); zhangml@hebut.edu.cn (M.Z.); srzhbgydx@163.com (R.S.) \\ * Correspondence: flyserelo@126.com
}

Citation: Pei, X.; Tian, Y.; Zhang, M.; Shi, R. The Boundary Proportion Differential Control Method of Micro-Deformable Manipulator with Compensator Based on Partial Differential Equation Dynamic Model. Micromachines 2021, 12, 799. https:// doi.org/10.3390/mi12070799

Academic Editor: Yangmin Li

Received: 31 May 2021

Accepted: 2 July 2021

Published: 5 July 2021

Publisher's Note: MDPI stays neutral with regard to jurisdictional claims in published maps and institutional affiliations.

Copyright: (c) 2021 by the authors. Licensee MDPI, Basel, Switzerland. This article is an open access article distributed under the terms and conditions of the Creative Commons Attribution (CC BY) license (https:// creativecommons.org/licenses/by/ $4.0 /)$.
Abstract: It is challenging to accurately judge the actual end position of the manipulator-regarded
as a rigid body-due to the influence of micro-deformation. Its precise and efficient control is a crucial
problem. To solve the problem, the Hamilton principle was used to establish the partial differential
equation (PDE) dynamic model of the manipulator system based on the infinite dimension of
the working environment interference and the manipulator space. Hence, it resolves the common
overflow instability problem in the micro-deformable manipulator system modeling. Furthermore,
an infinite-dimensional radial basis function neural network compensator suitable for the dynamic
model was proposed to compensate for boundary and uncertain external interference. Based on this
compensation method, a distributed boundary proportional differential control method was designed
to improve control accuracy and speed. The effectiveness of the proposed model and method was
verified by theoretical analysis, numerical simulation, and experimental verification. The results
show that the proposed method can effectively improve the response speed while ensuring accuracy.

Keywords: micro-deformable manipulator; partial differential equation dynamic model; radial basis function neural network compensator; boundary proportional differential control method

\section{Introduction}

With the rapid development of modern manipulators, the traditional rigid manipulators with large mass and margin cannot satisfy fast response and accurate positioning requirements. However, micro-deformable manipulators have lighter weight, lower energy consumption, and less inertia than traditional manipulators; moreover, they have high precision, high efficiency, high speed, high flexibility, high adaptability, and intelligence [1]. Their application range is wider than that of rigid manipulators. Therefore, the lightweight and dexterous micro-deformable manipulator dynamic model and its precise control have gradually become a hot research topic [2].

Researchers have done much work on the dynamic modeling and precise control of micro-deformable manipulators. In [3], to control a single-link flexible manipulator, a hybrid method combining sliding mode and $H-\infty$ theory was proposed. Furthermore, a linear optimal damping controller was used in [4] to adjust the flexible boom vibration mode to a lower level. Based on the partial differential equation (PDE) dynamic model of the flexible manipulator system, some researchers used the adaptive boundary control method [5-7] to control the manipulator, while others used the neural network control method [8]. Several researchers have combined the adaptive boundary control method with the approximation or compensation results of radial basis function (RBF) neural network [9-13] to optimize the control performance of flexible systems. Additionally, the RBF neural network proportional differential (PD) control method of the flexible manipulator was studied in [14]. Moreover, in [15], based on a wavelet neural network, a dynamic surface control method was proposed. Furthermore, the iterative learning-based adaptive control methods were designed in $[16,17]$ to obtain trajectory tracking and vibration 
reduction. The dynamic model of the flexible manipulator system was established, and the dynamic characteristics were analyzed in addition to different methods in [18-20]. In [21,22], the boundary control of flexible Timoshenko arm was studied and analyzed. Furthermore, the position control method and residual vibration of the flexible system were analyzed in [23-25]. Moreover, some optimized fuzzy control methods were proposed in [26-28]. However, most studies mentioned above simplified the micro-deformation manipulator into a relatively simple ideal model; these idealized models were not consistent with the actual situation $[6,8,17,23]$. Additionally, most studies ignored the infinite dimensionality of the micro-deformation manipulator. These lead to overflow and instability; moreover, change of the system space state cannot be described accurately $[18,19,26]$. The overflow problems were considered in a few PDE modeling papers $[5,7,9,12]$ and RBF neural network was used to estimate interference of one end, but the other end was ignored [9]. Due to the partial interference being neglected, the established model was not accurate enough. This study completely considered the interference at both ends of the boundary while establishing the PDE dynamic model. The estimated interference results were added to the control law using two outputs RBF neural network for improvement and optimization purposes. Thus, the accuracy of the model was improved, and the model fitted the actual situation better.

Furthermore, this study fully considered the vibration and deformation of the microdeformation manipulator and the joint micro-deformation. Moreover, we studied the distributed parameter boundary PD control method of RBF neural network compensator for the micro-deformation manipulator. First, the infinite-dimensional PDE dynamic model of the micro-deformable manipulator system was established. After that, the PD control method and stability analysis of the micro-deformable manipulator based on the RBF neural network compensator were introduced. Finally, numerical simulation and analysis of the control method were performed. Additionally, the response speed of the proposed method was increased by at least 30\% compared with the adaptive boundary control method and RBF neural network method. Experimental verification was carried out for the proposed method at last.

\section{Dynamic Modeling of Micro-Deformable Manipulator}

An accurate dynamic model is a basis for achieving high-performance control. The control methods of micro-deformation manipulators were developed based on the ordinary differential equation (ODE) dynamic model in most studies. However, it cannot accurately describe the distributed parameter characteristics of the micro-deformable structure and may cause overflow instability problems. The PDE dynamic model can reflect the dynamic characteristics of the micro-deformed structure more accurately than the ODE dynamic model $[5,9]$. The Hamilton method was used to derive the PDE dynamic equation of the micro-deformable manipulator system $[5,7,9,12]$; moreover, the corresponding boundary conditions of the system were obtained. This process did not require complex force analysis of the micro-deformable manipulator system. The dynamic model can be derived directly by mathematical methods.

Remark 1. The deformation of the micro-deformable manipulator is spatiotemporal, but the time variable $t$ does not affect the calculation and derivation of the dynamic model. For simplicity, we omitted the time variable, $t$, in the function variable. For example, $y(x, t)$ is expressed as $y(x)$, $l(x, t)$ is expressed as $l(x)$, and $\theta(t)$ is expressed as $\theta$.

Remark 2. The usage and explanation of subscripts in this paper are as follows:

$$
\begin{aligned}
& (*)_{x}=\frac{\partial(*)}{\partial x},(*)_{x x}=\frac{\partial^{2}(*)}{\partial x^{2}},(*)_{x x x}=\frac{\partial^{3}(*)}{\partial x^{3}},(*)_{x x x x}=\frac{\partial^{4}(*)}{\partial x^{4}},(*)_{t}=\frac{\partial(*)}{\partial t},(*)_{t t}=\frac{\partial^{2}(*)}{\partial t^{2}}, \\
& (*)_{x t t}=\frac{\partial(*)}{\partial x \partial t^{2}} .
\end{aligned}
$$

We considered the plane motion single-rod micro-deformable manipulator as the research object (Figure 1); its cross-section was circular, and its radius, R, was $0.01 \mathrm{~m}$. 
The relevant symbols of the manipulator are shown in Nomenclature. According to the theory and formulas of structural dynamics, mean normal stress ratio to shear stress can be calculated, as shown in Equation (1). Therefore, $\sigma_{\text {ave }} \gg \tau_{s}$. In other words, bending deformation is the main deformation; therefore, the shear deformation can be neglected. Hence, we developed the dynamic model based on the Euler-Bernoulli beam theory.

$$
\frac{\sigma_{\text {ave }}}{\tau_{s}}=\frac{\sigma_{\max } \cdot(2 / 3)}{\tau_{s}}=\frac{\left(M L y_{\max } / I_{z}\right) \cdot(2 / 3)}{M / A}=\frac{8 M / 3 \pi R^{3}}{M / \pi R^{2}}=\frac{8}{3 R} \approx 266.67
$$

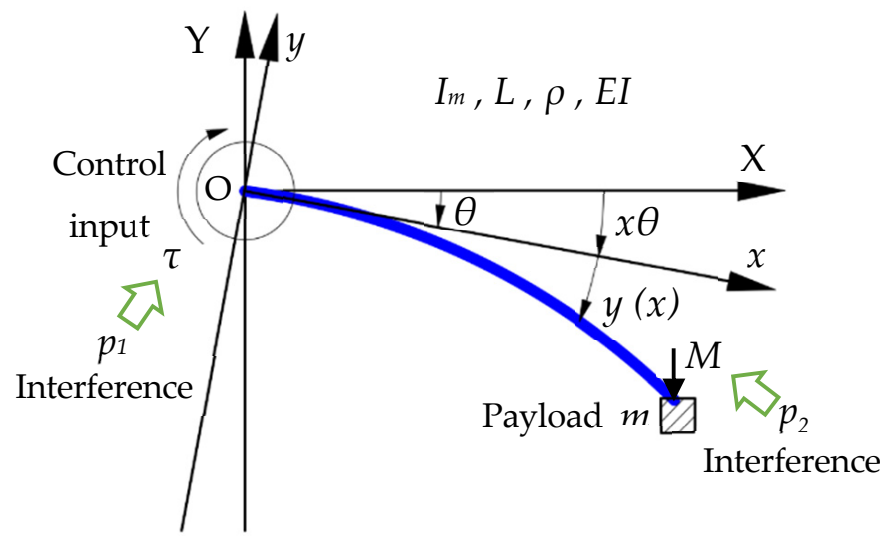

(a)

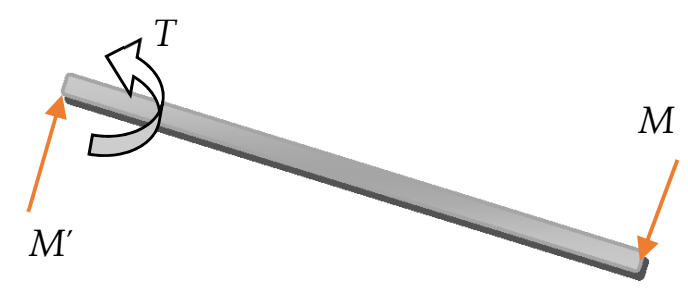

(b)

Figure 1. A typical single link micro-deformable manipulator: (a) coordinate diagram; (b) free-body diagram of the system.

The offset of any point, $[x, y(x)]$, in the follow-up coordinate system, $x O y$, from the micro-deformation manipulator in the inertial coordinate system, XOY, is approximately expressed as $l(x)$.

$$
l(x)=x \theta+y(x)
$$

The manipulator is regarded as an Euler-Bernoulli beam here and clamped to a motor at $x$. The natural boundary conditions can be expressed as:

$$
y(0)=y_{x}(0)=0
$$

Using Equations (2) and (3), we get:

$$
l(0)=0, l_{x}(0)=\theta, \frac{\partial^{m} l(x)}{\partial x^{m}}=\frac{\partial^{m} y(x)}{\partial x^{m}}, m \geq 2
$$

According to Hamilton principle $[5,6,29,30]$, the PDE dynamic equations of the microdeformable manipulator can be developed for every $0 \leq t_{1}<t<t_{2}$, as shown in Equation (5):

$$
\int_{t_{1}}^{t_{2}}\left(\delta W_{k}-\delta W_{p}+\delta W_{n c}\right) d t=0
$$

where $\delta W_{k}, \delta W_{p}$ and $\delta W_{n c}$ represent the kinetic energy, potential energy, and the variation of work done by non-conservative force, respectively; $t_{i}$ denotes a moment.

The total kinetic energy of the system can be obtained considering the rotational kinetic energy of the micro-deformation manipulator joint, the kinetic energy of the manipulator, and the kinetic energy of the load, as shown in Equation (6).

$$
W_{k}=\frac{1}{2} I_{m} \theta_{t}^{2}+\frac{1}{2} \int_{0}^{L} \rho l_{t}^{2}(x) d x+\frac{1}{2} m l_{t}^{2}(L)
$$


The potential energy of the system is expressed as:

$$
W_{p}=\frac{1}{2} \int_{0}^{L} E I y_{x x}^{2}(x) d x
$$

The work done by the non-conservative force of the system is expressed as:

$$
W_{n c}=\tau \theta+p_{1} \theta+M l(L)+p_{2} l(L)
$$

Substituting Equations (4), (6)-(8) into Equation (5), we get:

$$
\begin{aligned}
& \int_{t_{1}}^{t_{2}}\left(\delta W_{k}-\delta W_{p}+\delta W_{n c}\right) \\
& =-\int_{t_{1}}^{t_{2}} \int_{0}^{L} K_{1} \delta l(x) d x d t-\int_{t_{1}}^{t_{2}} K_{2} \delta l_{x}(0) d t-\int_{t_{1}}^{t_{2}} K_{3} \delta l(L) d t-\int_{t_{1}}^{t_{2}} K_{4} \delta l_{x}(L) d t=0
\end{aligned}
$$

where:

$$
\left\{\begin{array}{l}
K_{1}=\rho l_{t t}(x)+E I l_{x x x x}(x) \\
K_{2}=I_{m} l_{x t t}(0)-E I l_{x x}(0)-\left(\tau+p_{1}\right) \\
K_{3}=m l_{t t}(L)-E I l_{x x x}(L)-\left(M+p_{2}\right) \\
K_{4}=E I l_{x x}(L)
\end{array}\right.
$$

Each monomial in Equation (9) is linearly independent because $\delta l(x), \delta l_{x}(0), \delta l(L)$, $\delta l_{x}(L)$ are independent variables. Hence, $K_{1}=K_{2}=K_{3}=K_{4}=0$. Therefore, the PDE dynamic model of the micro-deformation manipulator system is obtained as Equation (10).

$$
\left\{\begin{array}{l}
\rho l_{t t}(x)=-E I l_{x x x x}(x) \\
\tau=I_{m} l_{x t t}(0)-E I l_{x x}(0)-p_{1} \\
M=m l_{t t}(L)-E I l_{x x x}(L)-p_{2} \\
l_{x x}(L)=0
\end{array}\right.
$$

where: $l_{t t}(x)=x \theta_{t t}+y_{t t}(x)$.

The dynamic model of the manipulator system was developed from the mathematical model perspective. The motion characteristics are related to time and position; therefore, the micro-deformable manipulator is essentially a distributed parameter system. Hence, the distributed parameter model was established based on the PDE equation. Furthermore, the corresponding control method adopted the distributed parameter boundary PD control, which can effectively obtain the micro-deformable system control. The boundary control only needs a small number of thrusters to achieve a better control effect than the discrete distributed control.

\section{RBF Neural Network Distributed Boundary PD Control Method}

The paper [5] proposed an adaptive boundary control method suitable for the PDE dynamic model. The method was simple and accurate, but the response time remained long. Compared with other machine learning algorithms, RBF neural network has the ability of parallel information processing, stronger computing power, and faster running speed. Moreover, RBF neural network can avoid the local minimum problem. Therefore, the control scheme based on RBF neural network is more suitable for the requirement of real-time control. Thus, RBF neural network compensator was used to improve the adaptive boundary control method described in [5]. The joint position was adjusted, and the vibration was weakened by designing the Lyapunov function and boundary PD control law (Figure 2). 


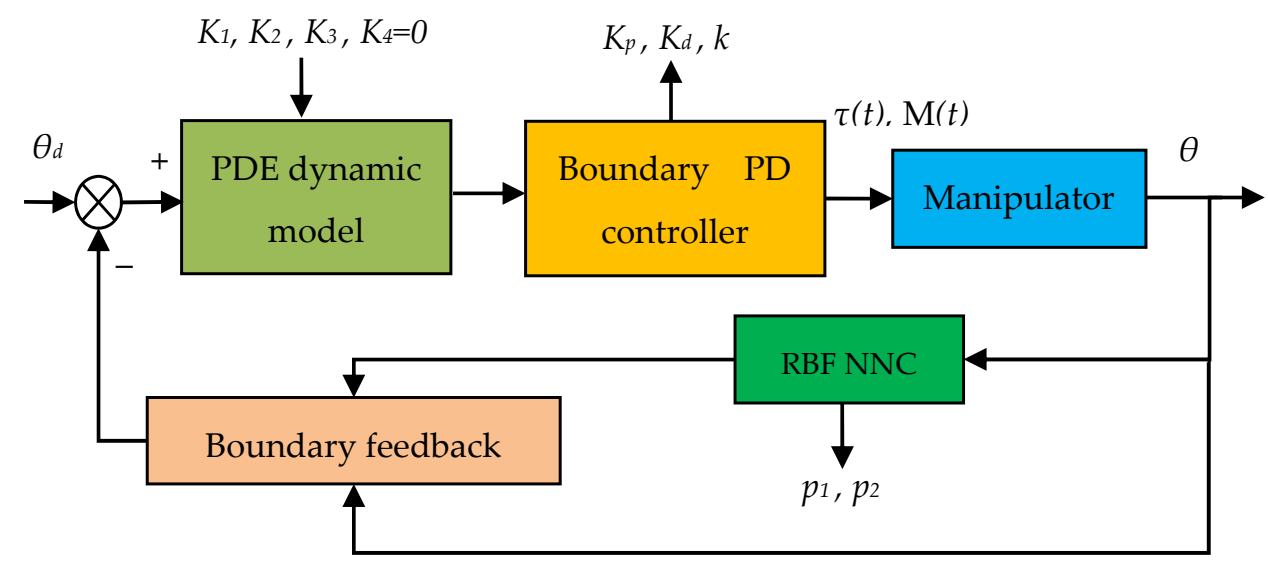

Figure 2. Boundary PD control principle with RBF neural network compensator.

\subsection{Design of Distributed Boundary PD Control Law Based on RBF Neural Network Compensator}

The error function is defined as $e=\theta-\theta_{d}$, where $\theta_{d}$ denotes the expectation angle. Consider that the uncertainty interference $p_{1}$ and $p_{2}$ of the actual model is unknown. The RBF neural network is used to estimate the uncertainty interference $p_{1}$ and $p_{2}$. The estimated values are $\hat{p}_{1}$ and $\hat{p}_{2}$. Furthermore, the input vector of the neural network is considered as $x=\left[\begin{array}{ccccc}e^{T} & \dot{e}^{T} & \theta_{d}^{T} & \dot{\theta}_{d}^{T} & \ddot{\theta}_{d}^{T}\end{array}\right]$. Moreover, the ideal RBF neural network algorithm is shown in Equation (11).

$$
\left\{\begin{array}{l}
\varphi_{i}=\exp \left(-\frac{\left\|x-c_{i}\right\|^{2}}{2 b_{i}^{2}}\right), i=1,2, \ldots, m \\
p_{1}=W_{1}^{*} \varphi_{1}(x)+\gamma_{1}, p_{2}=W_{2}^{*} \varphi_{2}(x)+\gamma_{2}
\end{array}\right.
$$

where $\varphi_{i}=\left[\varphi_{1}, \varphi_{2}, \ldots, \varphi_{m}\right]^{T}$ denotes the output vector of hidden layer obtained by Gaussian function, $m$ represents the number of neurons in the hidden layer, $c_{i}$ is the coordinate vector value of the Gaussian function's center point, $b_{i}$ represents the width of Gaussian function, $W_{i}^{*}$ denotes the ideal weight matrix of the neural network, and $\gamma_{i}$ is the network estimation error.

The output of the RBF neural network is shown in Equation (12).

$$
\left\{\begin{array}{l}
\hat{p}_{1}=\hat{W}_{1}^{*} \varphi_{1}(x) \\
\hat{p}_{2}=\hat{W}_{2}^{*} \varphi_{2}(x)
\end{array}\right.
$$

where $\hat{W}_{i}^{*}$ represents the estimated weight of the neural network for unknown parameter estimation.

The errors of the above estimation results are defined in Equation (13).

$$
\left\{\begin{array}{l}
\widetilde{W}_{1}^{*}=W_{1}^{*}-\hat{W}_{1}^{*}, \widetilde{W}_{2}^{*}=W_{2}^{*}-\hat{W}_{2}^{*} \\
\widetilde{p}_{1}=p_{1}-\hat{p}_{1}, \widetilde{p}_{2}=p_{2}-\hat{p}_{2}
\end{array}\right.
$$

Equations (11)-(13) suggest that

$$
\left\{\begin{array}{l}
\widetilde{p}_{1}=\widetilde{W}_{1}^{*} \varphi_{1}(x)+\gamma_{1} \\
\widetilde{p}_{2}=\widetilde{W}_{2}^{*} \varphi_{2}(x)+\gamma_{2}
\end{array}\right.
$$

Furthermore, the estimated results, $\hat{p}_{1}$ and $\hat{p}_{2}$, of the RBF neural network were added into the control law as a compensator to compensate for the external interference, in order to obtain the angular response of the micro-deformation manipulator and suppress its deformation and vibration effectively. The RBF-based boundary PD control law is presented as: 


$$
\left\{\begin{array}{l}
\tau=-k_{p} e-k_{d} e_{t}+\hat{p}_{1} \\
M=-k u_{\alpha}+m l_{x x x t}(L)+\hat{p}_{2}
\end{array}\right.
$$

where: $k_{p}, k_{d}$, and $k$ are control gain values and $k_{p}>0, k_{d}>0, k>0, u_{\alpha}=l_{t}(L)-l_{x x x}(L)$, $e_{t}=\theta_{t}, e_{t t}=\theta_{t t}$.

Remark 3. All parameters mentioned in the designed control law (Equation (15)) are measurable or computable. We can use the position sensor, tachometer, laser displacement sensor, and shear force sensor to get the measurements, $\theta, \theta_{t}, l(x)$, and $l_{x x x}(x)$, respectively. $e_{,} e_{t}, e_{t t}, l_{t}(x)$, and $l_{x x x t}(x)$ can be calculated from the measurements.

\subsection{Stability Analysis Based on Lyapunov Function}

Lemma 1. For $\phi_{1}(x, t), \phi_{2}(x, t) \in R, x \in[0, L], t \in[0, \infty)$, the following inequality holds:

$$
\begin{aligned}
& \phi_{1}(x, t) \phi_{2}(x, t) \leq\left|\phi_{1}(x, t) \phi_{2}(x, t)\right| \leq \phi_{1}{ }^{2}(x, t)+\phi_{2}{ }^{2}(x, t) \\
& \left|\phi_{1}(x, t) \phi_{2}(x, t)\right| \leq \frac{1}{\lambda} \phi_{1}{ }^{2}(x, t)+\lambda \phi_{2}{ }^{2}(x, t),(\lambda>0)
\end{aligned}
$$

Lemma 2. For $p(x, t) \in R, x \in[0, L], t \in[0, \infty)$, if $p(0, t)=0, t \in[0, \infty)$, then $p^{2}(x, t) \leq$ $L \int_{0}^{L} p_{x}^{2}(x, t) d x, x \in[0, L]$. Similarly, if $p_{x}(0, t)=0, t \in[0, \infty)$, then $p_{x}^{2}(x, t) \leq L \int_{0}^{L} p_{x x}^{2}(x, t) d x$, $x \in[0, L]$.

Lemma 3. For $V:[0, \infty) \in R, t \geq t_{0} \geq 0$, if $\dot{V} \leq-\eta V+g$, then:

$$
V(t) \leq e^{-\eta\left(t-t_{0}\right)} V\left(t_{0}\right)+\int_{t_{0}}^{t} e^{-\eta(t-s)} g(s) d s, \eta>0 .
$$

Theorem 1. The closed-loop system described by Equation (10) is asymptotically stable, with the proposed RBF neural network compensator (Equation (12)) and control law (Equation (15)), namely, $\theta \rightarrow \theta_{d}, \theta_{t} \rightarrow 0, y(x) \rightarrow 0, y_{t}(x) \rightarrow 0$, as $x \in[0, L], t \rightarrow \infty$.

Proof of Theorem 1. The Lyapunov function is defined to prove the stability of the controller, as in Equation (16).

$$
H(t)=W_{1}+W_{2}+W_{\alpha}
$$

where:

$$
\left\{\begin{array}{l}
W_{1}=\frac{1}{2} \int_{0}^{L} \rho l_{t}^{2}(x) d x+\frac{1}{2} E I \int_{0}^{L} y_{x x}^{2}(x) \\
W_{2}=\frac{1}{2} I_{m} e_{t}^{2}+\frac{1}{2} k_{p} e^{2}+\frac{1}{2} m u_{\alpha}^{2}-p_{1} e-p_{2} \int_{t_{1}}^{t_{2}} u_{\alpha} d t \\
W_{\alpha}=\alpha \rho \int_{0}^{L} x l_{t}(x) l e_{x}(x) d x+\alpha I_{m} e e_{t}
\end{array}\right.
$$

In Equation (17), $W_{1}$ represents the inhibition index bending deformation of the microdeformation manipulator. The first two items in $W_{2}$ represent the control error-index, and the rest of the items are the auxiliary terms. Moreover, $W_{\alpha}$ is the auxiliary part and $\alpha$ denotes a small positive constant. Furthermore,

$$
l e(x)=x e+y(x), l e_{x}(x)=e+y_{x}(x), l e_{x x}(x)=y_{x x}(x)=l_{x x}(x)
$$

According to Lemmas 1 and 2, we obtain:

$$
\begin{aligned}
& \left|W_{\alpha}\right| \leq \alpha \rho L \int_{0}^{L} l_{t}^{2}(x) d x+2 \alpha \rho L^{2} e^{2}+2 \alpha \rho L^{3} \int_{0}^{L} l_{x x}^{2}(x) d x+\alpha I_{m}\left(e^{2}+e_{t}^{2}\right) \\
& \leq \alpha_{1}\left(W_{1}+W_{2}\right)
\end{aligned}
$$

where: $\alpha_{1}=\max \left(2 \alpha L, 2 \alpha \rho L^{3} / E I, 2\left(\alpha I_{m}+2 \alpha \rho L^{2}\right) / k_{p}, 2 \alpha\right)$. 
Therefore,

$$
-\alpha_{1}\left(W_{1}+W_{2}\right) \leq W_{\alpha} \leq \alpha_{1}\left(W_{1}+W_{2}\right)
$$

Take $0<\alpha_{1}<1$, i.e., $0<\max \left(2 \alpha L, 2 \alpha \rho L^{3} / E I, 2\left(\alpha I_{m}+2 \alpha \rho L^{2}\right) / k_{p}, 2 \alpha\right)<1$, then $\alpha$ can be designed as $0<\alpha<\frac{1}{\left(2 L, 2 \rho L^{3} / E I, 2\left(I_{m}+2 \rho L^{2}\right) / k_{p}, 2\right)}$. We define $1>\alpha_{2}=1-\alpha_{1}>0$, $2>\alpha_{3}=1+\alpha_{1}>1$; hence,

$$
0 \leq \alpha_{2}\left(W_{1}+W_{2}\right) \leq H(t) \leq \alpha_{3}\left(W_{1}+W_{2}\right)
$$

According to inequality in Equation (21), the Lyapunov function is positively definite. Hence,

$$
H_{t}(t)=\left(W_{1}\right)_{t}+\left(W_{2}\right)_{t}+\left(W_{\alpha}\right)_{t}
$$

In Equation (22),

$$
\left\{\begin{array}{l}
\left(W_{1}\right)_{t}=\int_{0}^{L} \rho l_{t}(x) l_{t t}(x) d x+E I \int_{0}^{L} y_{x x}(x) y_{x x t}(x) \\
\left(W_{2}\right)_{t}=I_{m} e_{t} e_{t t}+k_{p} e e_{t}+m u_{\alpha}\left(u_{\alpha}\right)_{t}-p_{1} e_{t}-p_{2} u_{\alpha} \\
\left(W_{\alpha}\right)_{t}=\left(W_{\alpha 1}\right)_{t}+\left(W_{\alpha 2}\right)_{t}+\left(W_{\alpha 3}\right)_{t}
\end{array}\right.
$$

In Equation (23),

$$
\left\{\begin{array}{l}
\left(W_{\alpha 1}\right)_{t}=\alpha \rho \int_{0}^{L} x l_{t t}(x) l e_{x}(x) d x \\
\left(W_{\alpha 2}\right)_{t}=\alpha \rho \int_{0}^{L} x l_{t}(x) l_{t} e_{x}(x) d x \\
\left(W_{\alpha 3}\right)_{t}=\alpha I_{m}\left(e_{t}^{2}+e e_{t t}\right)
\end{array}\right.
$$

Substituting Equation (10) into Equation (23) and combining Equation (4), $\left(W_{1}\right)_{t}$ can be rewritten as:

$$
\left(W_{1}\right)_{t}=-E I y_{x x x}(L) l_{t}(L)-E I y_{x x}(0) \theta_{t}=-E I l_{x x}(0) e_{t}-E I l_{x x x}^{2}(L)-E I l_{x x x}(L) u_{\alpha}
$$

Using Equations (10) and (15) and combining Equations (23) and (25), we obtain:

$$
\begin{aligned}
& \left(W_{1}\right)_{t}+\left(W_{2}\right)_{t}=-E I l_{x x}(0) e_{t}-E I l_{x x x}^{2}(L)-E I l_{x x x}(L) u_{\alpha}+I_{m} e_{t} e_{t t}+k_{p} e e_{t}+m u_{\alpha}\left(u_{\alpha}\right)_{t}-p_{1} e_{t}-p_{2} u_{\alpha} \\
& =e_{t}\left(-E I l_{x x}(0)+I_{m} e_{t t}+k_{p} e-p_{1}\right)+u_{\alpha}\left(-E I l_{x x x}(L)+m\left(u_{\alpha}\right)_{t}-p_{2}\right)-E I l_{x x x}^{2}(L) \\
& =e_{t}\left(\tau+k_{p} e-p_{1}\right)+u_{\alpha}\left(M-m l_{x x x t}(L)-p_{2}\right)-E I l_{x x x}^{2}(L) \\
& =-k_{d} e_{t}^{2}-k u_{\alpha}^{2}-E I l_{x x x}^{2}(L)
\end{aligned}
$$

According to Lemmas 1-3, combining Equations (10) and (18), and using the method of integral by parts, we deduce:

$$
\begin{gathered}
\left(W_{\alpha 1}\right)_{t}=-\alpha E I L l e_{x}(L) l_{x x x}(L)-\frac{3}{2} \alpha E I \int_{0}^{L} l_{x x}^{2}(x) d x-\alpha E I e l_{x x}(0) \\
\leq-\left(\frac{3}{2} \alpha-2 \alpha L^{2}-\frac{2 \alpha L^{3}}{E I}\right) \int_{0}^{L} E I l_{x x}^{2}(x) d x+\alpha E I L l_{x x x}^{2}(L)-\alpha E I e l_{x x}(0)+\left(2 \alpha E I L+2 \alpha L^{2}\right) e^{2} \\
\left(W_{\alpha 2}\right)_{t}=\frac{1}{2} \alpha \rho L l_{t}^{2}(L)-\frac{1}{2} \alpha \rho \int_{0}^{L} l_{t}^{2}(x) d x \\
\left(W_{\alpha 3}\right)_{t}=\alpha I_{m} e_{t}^{2}+\alpha e E I l_{x x}(0)-\alpha k_{p} e^{2}-k_{d} \alpha e e_{t} \leq\left(\alpha I_{m}+k_{d} \alpha\right) e_{t}^{2}-\left(\alpha k_{p}-k_{d} \alpha\right) e^{2}+\alpha e E I l_{x x}(0)
\end{gathered}
$$

We obtain Equation (30) using Equations (23), (27)-(29).

$$
\begin{aligned}
& \left(W_{\alpha}\right)_{t} \leq-\left(\frac{3}{2} \alpha-2 \alpha L^{2}-\frac{2 \alpha L^{3}}{E I}\right) \int_{0}^{L} E I l_{x x}^{2}(x) d x+\alpha E I L l_{x x x}^{2}(L)+\left(2 \alpha E I L+2 \alpha L^{2}\right) e^{2} \\
& +\frac{1}{2} \alpha \rho L l_{t}^{2}(L)-\frac{1}{2} \alpha \rho \int_{0}^{L} l_{t}^{2}(x) d x+\left(\alpha I_{m}+k_{d} \alpha\right) e_{t}^{2}-\left(\alpha k_{p}-k_{d} \alpha\right) e^{2}
\end{aligned}
$$


Substituting Equations (26) and (30) into Equation (22), we get Equation (31).

$$
\begin{aligned}
& H_{t}(t)=\left(W_{1}\right)_{t}+\left(W_{2}\right)_{t}+\left(W_{\alpha}\right)_{t} \\
& \leq-k_{d} e_{t}^{2}-k u_{\alpha}^{2}-E I l_{x x x}^{2}(L)-\left(\frac{3}{2} \alpha-2 \alpha L^{2}-\frac{2 \alpha L^{3}}{E I}\right) \int_{0}^{L} E I l_{x x}^{2}(x) d x+\alpha E I L l_{x x x}^{2}(L) \\
& +\left(2 \alpha E I L+2 \alpha L^{2}\right) e^{2}+\frac{1}{2} \alpha \rho L l_{t}^{2}(L)-\frac{1}{2} \alpha \rho \int_{0}^{L} l_{t}^{2}(x) d x+\left(\alpha I_{m}+k_{d} \alpha\right) e_{t}^{2}-\left(\alpha k_{p}-k_{d} \alpha\right) e^{2} \\
& =-\left(\frac{3}{2} \alpha-2 \alpha L^{2}-\frac{2 \alpha L^{3}}{E I}\right) \int_{0}^{L} E I l_{x x}^{2}(x) d x-\frac{1}{2} \alpha \int_{0}^{L} \rho l_{t}^{2}(x) d x-\left(k_{d}-\alpha I_{m}-k_{d} \alpha\right) e_{t}^{2} \\
& -\left(\alpha k_{p}-k_{d} \alpha-2 \alpha E I L-2 \alpha L^{2}\right) e^{2}-k u_{\alpha}^{2}+\frac{1}{2} \alpha \rho L l_{t}^{2}(L)-(E I-\alpha E I L) l_{x x x}^{2}(L)
\end{aligned}
$$
tees that:

The inequality $E I-\alpha E I L>\frac{1}{2} \alpha \rho L$ can be established by choosing $\alpha$, which guaran-

$$
\frac{1}{2} \alpha \rho L l_{t}^{2}(L)-(E I-\alpha E I L) l_{x x x}^{2}(L) \leq \eta_{0}\left(l_{t}(L)-l_{x x x}(L)\right)^{2}=\eta_{0} u_{\alpha}^{2}
$$

where: $\eta_{0}>\max \left(\eta_{1}, \frac{\eta_{1} \eta_{2}}{\eta_{2}-\eta_{1}}\right), \eta_{1}=\frac{1}{2} \alpha \rho L, \eta_{2}=E I-\alpha E I L$.

According to Equations (31) and (32), we obtain Equation (33).

$$
\begin{aligned}
& H_{t}(t) \leq-\left(\frac{3}{2} \alpha-2 \alpha L^{2}-\frac{2 \alpha L^{3}}{E I}\right) \int_{0}^{L} E I l_{x x}^{2}(x) d x-\frac{1}{2} \alpha \int_{0}^{L} \rho l_{t}^{2}(x) d x-\left(k_{d}-\alpha I_{m}-k_{d} \alpha\right) e_{t}^{2} \\
& -\left(\alpha k_{p}-k_{d} \alpha-2 \alpha E I L-2 \alpha L^{2}\right) e^{2}-\left(k-\eta_{0}\right) u_{\alpha}^{2} \leq-\lambda_{0}\left(W_{1}+W_{2}\right) \leq-\lambda_{0} \frac{H(t)}{\alpha_{3}}=-\lambda H(t)
\end{aligned}
$$

The following constraints must be met to ensure the validity of Equation (33).

$$
\begin{aligned}
& \varepsilon_{1}=\frac{3}{2} \alpha-2 \alpha L^{2}-\frac{2 \alpha L^{3}}{E I}>0, \varepsilon_{2}=\frac{1}{2} \alpha>0, \varepsilon_{3}=k_{d}-\alpha I_{m}-k_{d} \alpha>0, \\
& \varepsilon_{4}=\alpha k_{p}-k_{d} \alpha-2 \alpha E I L-2 \alpha L^{2}>0, \varepsilon_{5}=k-\eta_{0}>0, \min \left(2 \varepsilon_{1}, 2 \varepsilon_{2}, \frac{2 \varepsilon_{3}}{I_{m}}, \frac{2 \varepsilon_{4}}{k_{p}}, \frac{2 \varepsilon_{5}}{m}\right) \geq \lambda_{0}>0, \lambda=\frac{\lambda_{0}}{\alpha_{3}}
\end{aligned}
$$

The solution for the inequality in Equation (33) is $H(t) \leq H(0) e^{-\lambda t}$. Therefore, the Lyapunov function $H(t)$ is close to 0 if the condition $H(0)$ is bounded.

According to Equation (21), $W_{1}+W_{2} \rightarrow 0$, therefore, $e \rightarrow 0$ and $e_{t} \rightarrow 0$, meaning $\theta \rightarrow \theta_{d}$ and $\theta_{t} \rightarrow 0$. Additionally, $l_{t}(x) \rightarrow 0, y_{t}(x) \rightarrow 0$. Moreover, according to Lemma $2, y_{x}^{2}(x) \leq L \int_{0}^{L} y_{x x}^{2}(x) d x, y^{2}(x) \leq L \int_{0}^{L} y_{x}^{2}(x) d x$. Therefore, we can further deduce that $y(x) \rightarrow 0$. Moreover, the closed-loop system tends to be stable.

\section{Numerical Simulation Analysis}

Three simulation tests were performed to verify the performance and effect of the proposed control method. During simulation, the discrete time was $\Delta t=5 \times 10^{-4}$ and discrete distance was $\Delta x=0.01 \mathrm{~m}$. The system parameters and controller coefficients were set as shown in Table 1. The initial state of the system and the initial interference compensation were set to 0 . The number of neurons in the input layer, hidden layer, and output layer of the RBF neural network was 5, 5, and 2, respectively. According to the actual range of the input layer $x$, the parameters of the Gaussian basis function of the hidden layer were set to: $c_{j}=\left[\begin{array}{ccccc}-2.5 & -1.5 & 0 & 1.5 & 2.5 \\ -2 & -1 & 0 & 1 & 2 \\ -1.5 & -0.75 & 0 & 0.75 & 1.5 \\ -1 & -0.5 & 0 & 0.5 & 1 \\ -0.5 & -0.25 & 0 & 0.25 & 0.5\end{array}\right], b_{j}=0.5$. In order to ensure reasonable results, the parameters and coefficients in the simulation scenarios 1-3 should be consistent.

Scenario 1: with the adaptive boundary control method [5].

Scenario 2: with the RBF neural network control method [8].

Scenario 3: with the proposed control method. 
Table 1. Numerical simulation marks of micro-deformable manipulator system.

\begin{tabular}{cccccccccc}
\hline Mark & $\boldsymbol{E I}$ & $\boldsymbol{m}$ & $\boldsymbol{I}_{\boldsymbol{m}}$ & $\boldsymbol{L}$ & $\boldsymbol{\rho}$ & $\boldsymbol{k}_{\boldsymbol{p}}$ & $\boldsymbol{k}_{\boldsymbol{d}}$ & $\boldsymbol{k}$ & $\boldsymbol{\lambda}$ \\
\hline Value & 3 & 0.15 & 0.1 & 1.0 & 0.21 & 60 & 30 & 20 & 200 \\
Unit & $\mathrm{N} \cdot \mathrm{m}^{2}$ & $\mathrm{~kg}$ & $\mathrm{~kg} \cdot \mathrm{m}^{2}$ & $\mathrm{~m}$ & $\mathrm{~kg} / \mathrm{m}$ & $/$ & $/$ & $/$ & $/$ \\
\hline
\end{tabular}

Remark 4 . The design description of coefficients $k_{p}, k_{d}$, and in control law is shown in Appendix A.

Remark 5. An appropriately large control gain $k_{p}$ can ensure the good tracking performance of the system, but if too large, the system will become invalid. Therefore, in practical operation, the system performance and the actuator saturation should be considered simultaneously when we design the coefficient.

The simulation results of the micro-deformable manipulator with the proposed control method are shown in Figures 3c, 4, 5c, 6c and 7c; results with the adaptive boundary control method are shown in Figures 3a, 5a, 6a and 7a; Figures 3b, 5b, 6b and 7b show the simulation results with the RBF neural network control method.

Figure 3 shows the angle tracking and angular velocity response results of microdeformable manipulator with different methods. As shown in Figure 3a,b, the adaptive boundary control method and RBF neural network control method affect the control performance due to the large error and long response time. On the contrary, the proposed control method can accurately adjust the joint angle to the expected value within $6 \mathrm{~s}$, as shown in Figure 3c. The response time comparisons of three methods are shown in Table 2. As shown in Table 2, the response speed is improved by at least $30 \%$ than that of the other two methods. Therefore, the compensation results of the RBF neural network are satisfactory, as shown in Figure 4.
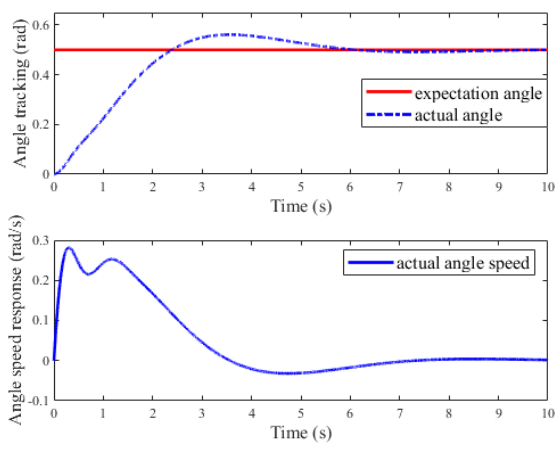

(a)
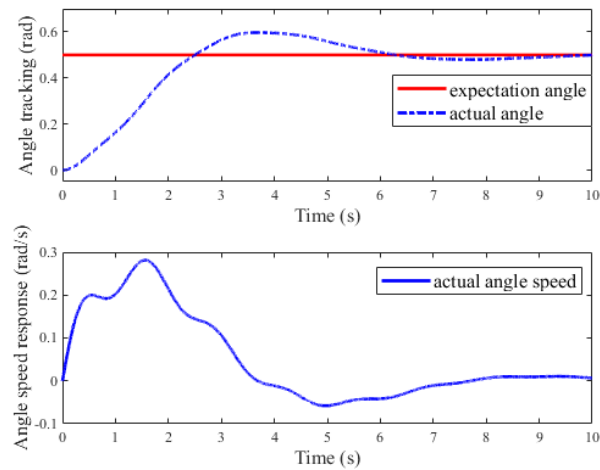

(b)
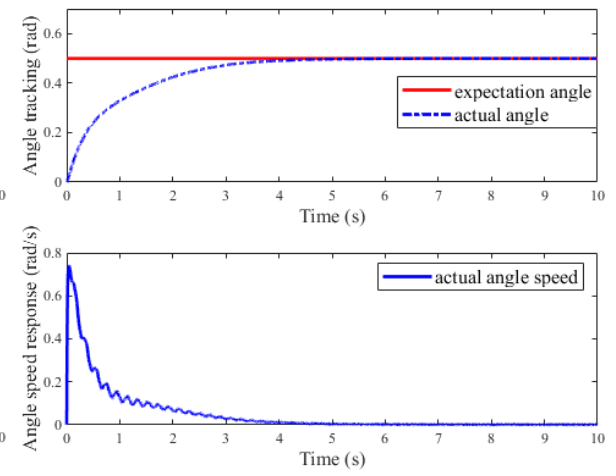

(c)

Figure 3. The angle tracking and response results of different methods: (a) adaptive boundary method; (b) RBF neural network method; (c) the proposed method.

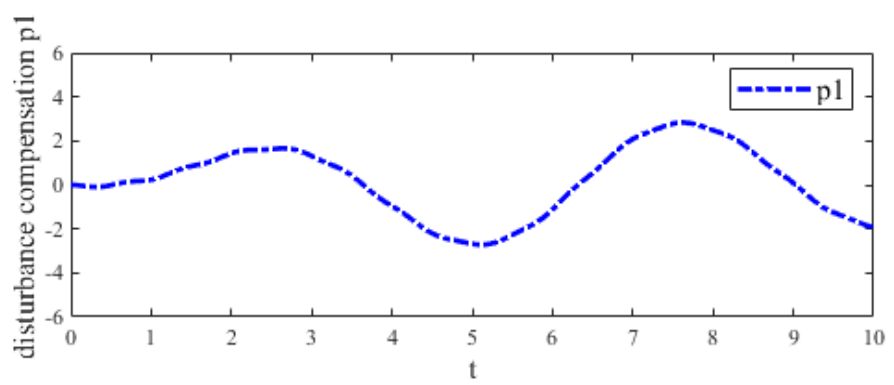

(a)

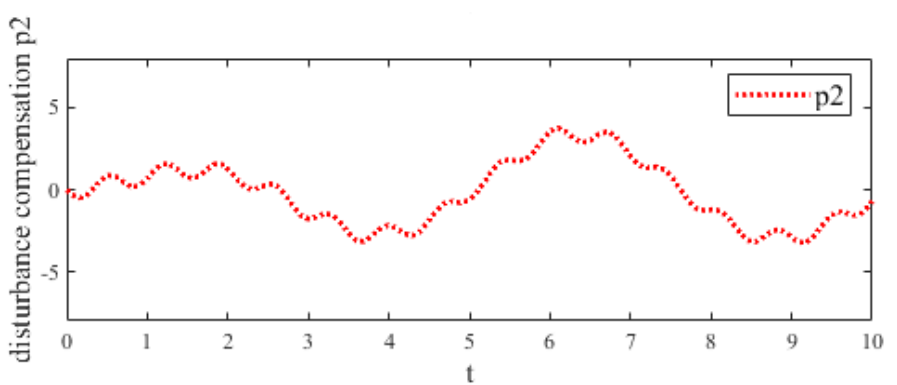

(b)

Figure 4. RBF neural network compensation results: (a) compensation results of $p_{1}$; (b) compensation results of $p_{2}$. 
Figures 5 and 6 show the elastic deformation and deformation rate of manipulator successively. The statistical results of deformation rate are shown in Table 2. As shown in Figure 5a,b, the elastic deformation of the micro-deformable manipulator is remarkable, and it is not suppressed obviously. Conversely, in Figure $5 c$, the elastic deformation reaches the peak in $5 \mathrm{~s}$ and is eliminated obviously in $7 \mathrm{~s}$. Furthermore, compared with Scenarios 1 and 2, the deformation rate of the manipulator is reduced by an order of magnitude with the proposed method (Table 2).

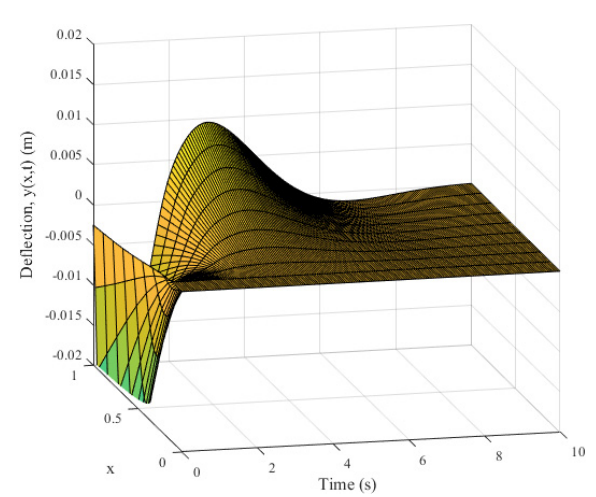

(a)

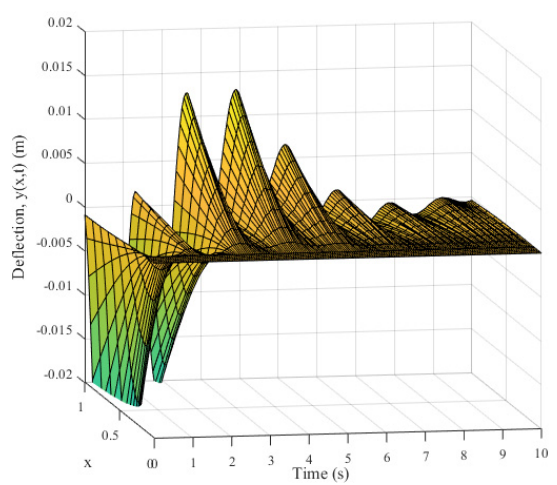

(b)

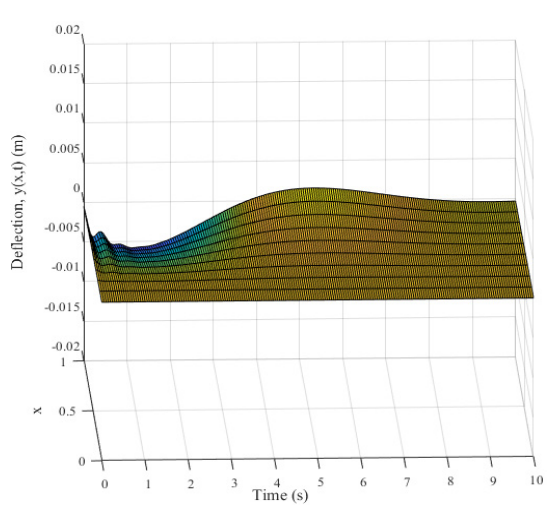

(c)

Figure 5. The deformation results of different methods: (a) adaptive boundary method; (b) RBF neural network method; (c) the proposed method.

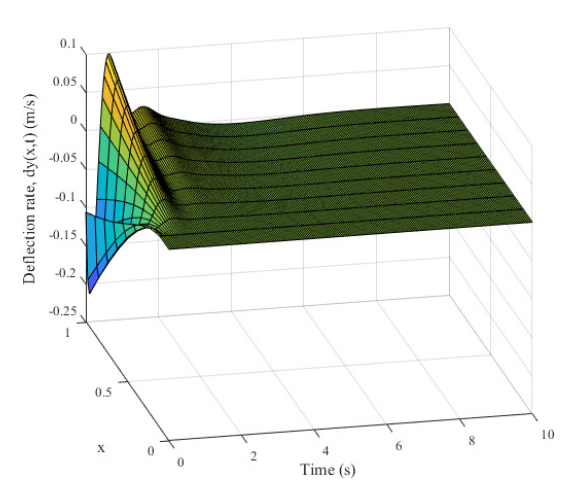

(a)

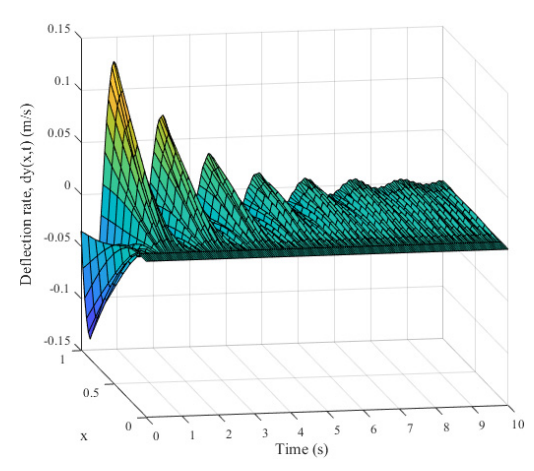

(b)

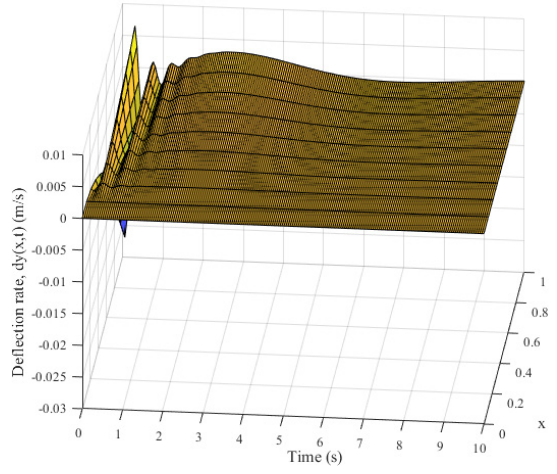

(c)

Figure 6. The deformation rate results of different methods: (a) adaptive boundary method; (b) RBF neural network method; (c) the proposed method.

Figure 7 depicts the corresponding control input with different methods. Furthermore, the related statistical results are shown in Table 2. In Figure 7a,b, the control input of the adaptive boundary control method and RBF neural network control method is unacceptable because of sizeable fluctuation and overshoot. Conversely, in Figure 7c, the fluctuation and overshoot are nearly weakened within $2 \mathrm{~s}$.

Table 2. The statistical results of the simulation data of the proposed method and the comparison methods.

\begin{tabular}{cccc}
\hline Comparisons & Adaptive Boundary Method & RBF Neural Network Method & The Proposed Method \\
\hline Response time $(\mathrm{s})$ & 7 & 9 & 6 \\
Maximum of & -0.2132 & -0.1396 & -0.0269 \\
deformation rate $(\mathrm{m} / \mathrm{s})$ & 0.2964 & 0.7233 & 0.3333 \\
Mean value of $\mathrm{M}(\mathrm{N} \cdot \mathrm{m})$ & -0.3334 & -0.8040 & -0.3750 \\
Mean value of $\tau(\mathrm{N} \cdot \mathrm{m})$ & & & \\
\hline
\end{tabular}




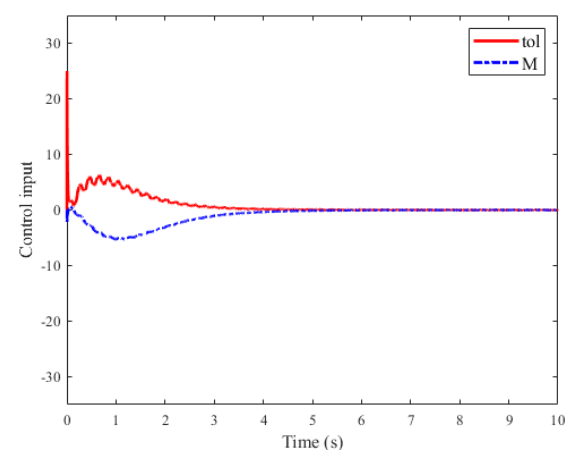

(a)

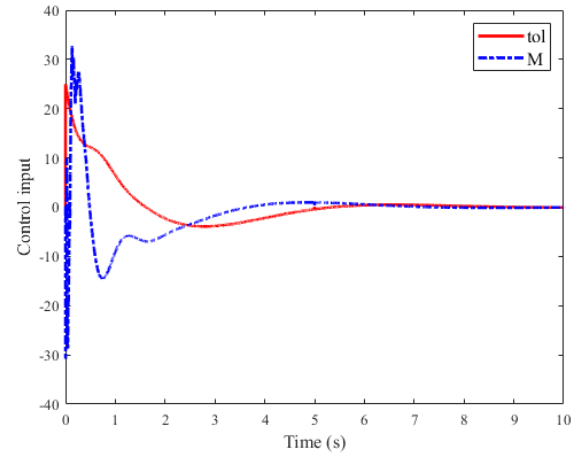

(b)

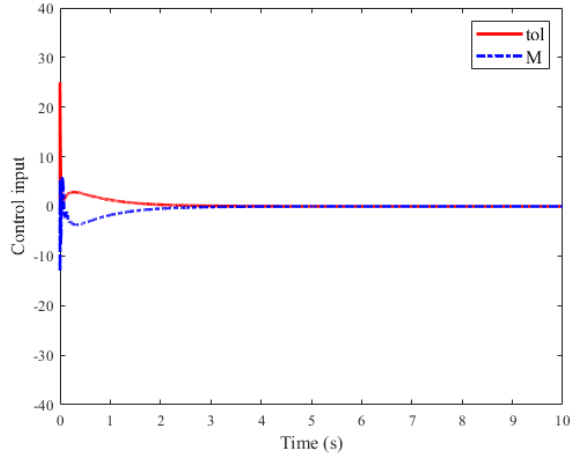

(c)

Figure 7. The control input $\tau$ and $M$ results of different methods: (a) adaptive boundary method; (b) RBF neural network method; (c) the proposed method.

The three simulations confirmed that the proposed method performs excellently in trajectory tracking and vibration and deformation suppression of the micro-deformable manipulator under the premise of considering the double boundary interference. Through the analysis of the above simulation results, the proposed method is superior to the adaptive boundary control method and RBF neural network control method. Compared with the other two methods, the response time of the proposed method is reduced by at least $30 \%$. The deformation of the manipulator is restrained to a great extent.

\section{Experimental Tests}

A simple slender single link micro-deformable manipulator $(\mathrm{L}=1 \mathrm{~m}, \mathrm{R}=0.01 \mathrm{~m}$ which are consistent with the numerical analysis) was used for the experimental test in this paper. Air experiment was carried out in the laboratory. The experimental apparatus is shown in Figure 8a. In each experiment, the real-time position, current, speed, and other information of the micro-deformable manipulator were sent to the computer through the serial port, and then the computer, as the host computer, sent the order to the driver which drives the thruster to control the motion of the manipulator. The schematic diagram of the experiment is shown in Figure $8 \mathrm{~b}$. The thruster here was a MOTEC DC servo motor of DSEM-V241230E60LN type. It was driven by MOTEC DC servo driver of ARES-80-15-E-A0 type. The host computer here was a notebook computer with Intel Core i7-5500u $2.5 \mathrm{GHz}$ CPU and 12 GB of RAM, running under Windows 10 operating system. The control software was developed by Visual Studio 2017. The manipulator system was powered by 24 V DC power supply.

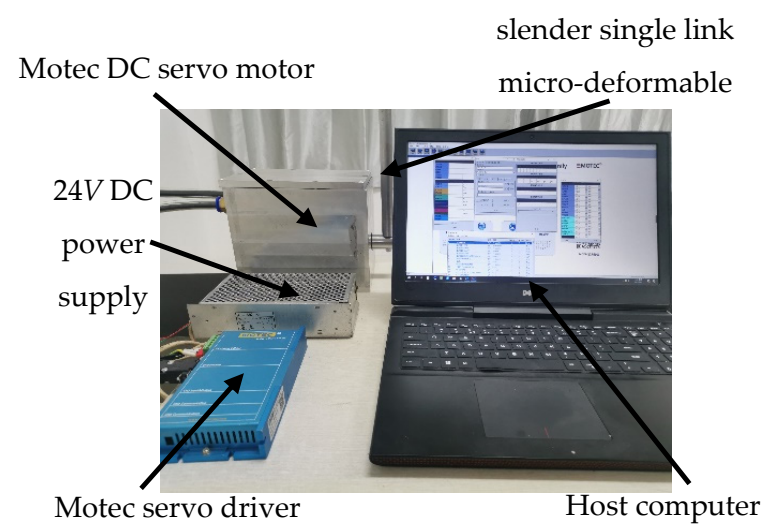

(a)

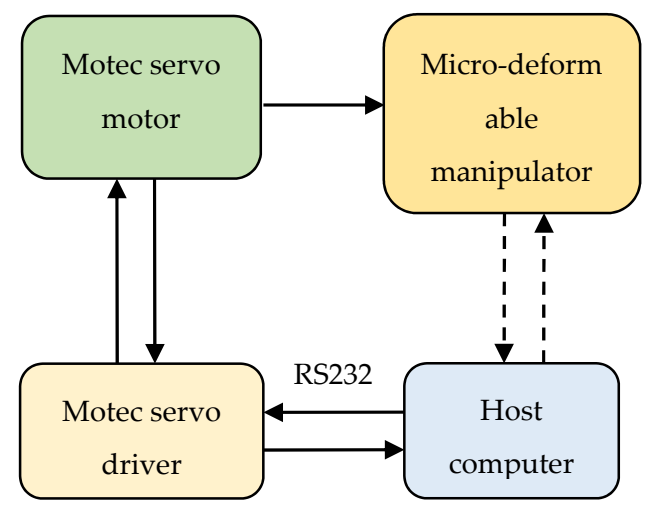

(b)

Figure 8. Experimental apparatus and schematic diagram: (a) experimental apparatus of the micro-deformable manipulator; (b) the schematic diagram of the experiment. 
In each air experiment, the parameters of the motor were set to pulse $/ 2 \pi \mathrm{rad}$ in advance. The manipulator was controlled to rotate 1592 pulses per time, which was about $0.5 \mathrm{rad}$, and swing back and forth every time. In order to ensure the accuracy of data analysis, the angle set in the experiment was consistent with that in the simulation. The data of one cycle were collected for analysis (Figure 9).

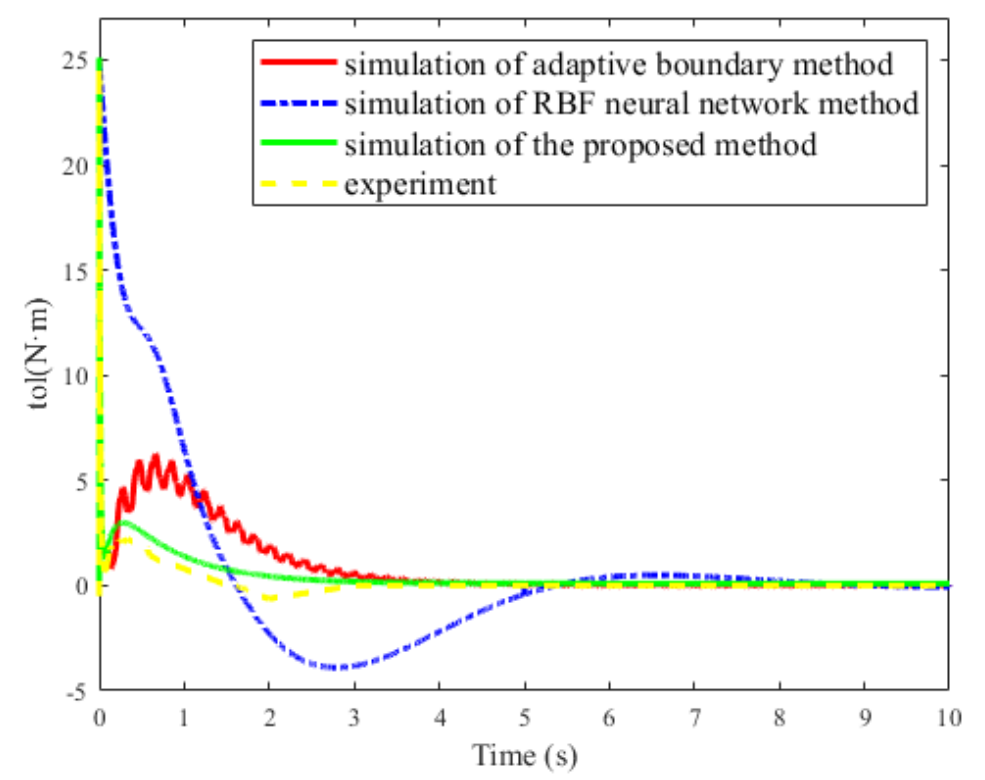

(a)

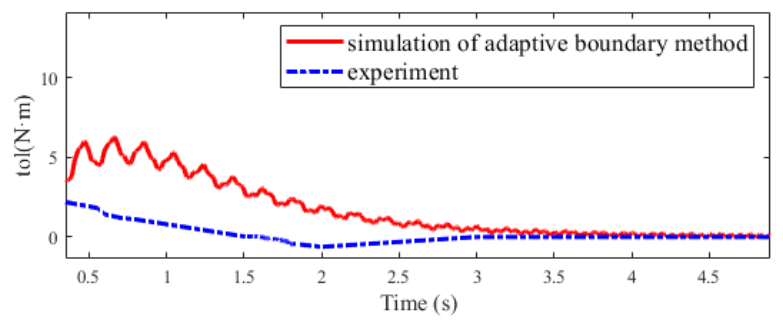

(b)

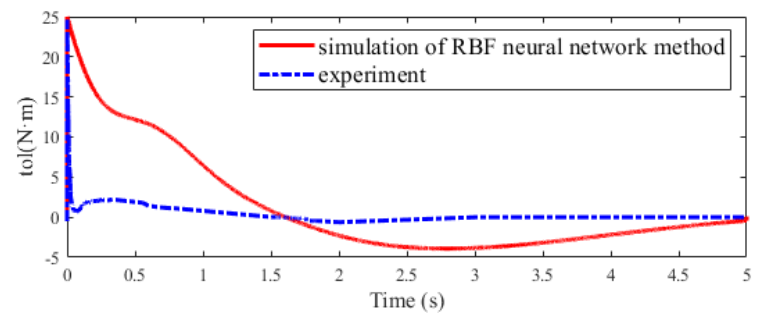

(c)

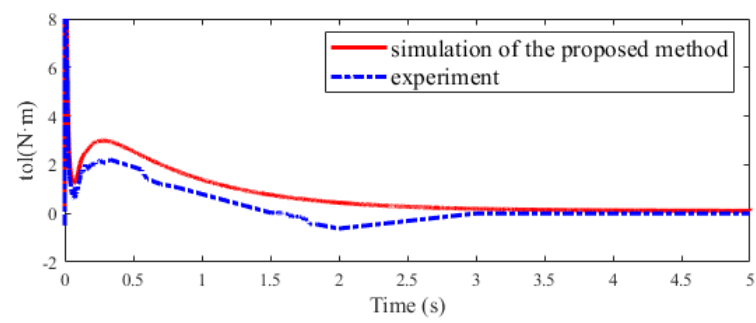

(d)

Figure 9. Comparison of $\tau$ between experiment and simulations: (a) comparison of overall results; (b) comparison of partial enlarged results of adaptive boundary method; (c) comparison of partial enlarged results of the RBF neural network method; (d) comparison of partial enlarged results of the proposed method.

Figure 9 depicts the change of the torque values of those collected from the experiment and simulated with different methods. The errors of experiment and three simulations are recorded in Table 3. In Figure 9b,c, the other two methods are not satisfactory due to their sizeable errors. As shown in Figure 9d, the variation of the numerical torque value of the proposed method is similar to that of the experimental torque value. The maximum error did not exceed $0.7 \mathrm{~N} \cdot \mathrm{m}$ as shown in Table 3, which verified the correctness of the numerical results of the proposed method.

Table 3. The errors between experiment and three simulations.

\begin{tabular}{cccc}
\hline Errors (N·m) & Adaptive Boundary Method & RBF Neural Network Method & The Proposed Method \\
\hline Mean value & 1.3190 & 6.6750 & 0.3008 \\
Standard deviation & 0.9203 & 8.5060 & 0.1604 \\
Maximum & 1.7620 & 20.930 & 0.6644 \\
\hline
\end{tabular}




\section{Conclusions}

This paper studied the PDE dynamic model; based on this model, the distributed boundary PD controller with infinite-dimensional RBF neural network compensator was proposed. The RBF neural network was used to compensate for double boundary interference and uncertain interference. The boundary control can be used to obtain the angle tracking of the micro-deformable manipulator. At the same time, it effectively weakened the vibration generated by the micro-deformation manipulator during the movement. The response time of the proposed method was reduced by at least $30 \%$ compared with the adaptive boundary controller and the RBF neural network controller. The error between the simulated results and experimental results was no more than $0.7 \mathrm{~N} \cdot \mathrm{m}$, which verified the accuracy of numerical results and the feasibility of the proposed method. Since the unknown interference distribution in the external space is random and unpredictable, it is essential to subdivide the spatially distributed interference further and study the corresponding compensation control method in the future.

Author Contributions: Conceptualization, X.P. and Y.T.; methodology, X.P.; software, X.P. and R.S.; validation, X.P., Y.T. and R.S.; formal analysis, X.P.; investigation, X.P. and M.Z.; resources, X.P. and M.Z.; data curation, R.S.; writing—original draft preparation, X.P.; writing-review and editing, Y.T.; visualization, X.P.; supervision, M.Z.; project administration, M.Z.; funding acquisition, M.Z. All authors have read and agreed to the published version of the manuscript.

Funding: This research is funded by the National key R\&D project of China (grant number: 2018YFB 1309400 and 2018YFB1309401), the Science and Technology Research Project of Hebei Province (grant number QN2018090) and the Natural Science Foundation of Hebei Province (grant number E2021202032).

Acknowledgments: The computational work and experimental test for this research were performed at the Special Robot Laboratory of Hebei University of Technology, China.

Conflicts of Interest: We state that we do not use this work commercially and have not published this article. The authors declare no conflict of interest.

\section{Nomenclature}

L Length of micro-deformable manipulator, $\mathrm{m}$

$R \quad$ Radius of micro-deformable manipulator, $\mathrm{m}$

$\rho \quad$ Linear density of micro-deformable manipulator, $\mathrm{kg} / \mathrm{m}$

$I_{m} \quad$ Center moment of inertia, $\mathrm{kg} \cdot \mathrm{m}^{2}$

EI Bending stiffness of uniform beam, $\mathrm{N} \cdot \mathrm{m}^{2}$

$\tau \quad$ Motor control input torque at initial end point, $\mathrm{N} \cdot \mathrm{m}$

$M \quad$ Control input force of end load, $\mathrm{N}$

$m \quad$ Terminal load mass, $\mathrm{kg}$

$\theta \quad$ Joint turning angle (excluding deformation),

$y(x) \quad$ Elastic deformation of manipulator at point $x, \mathrm{~m}$

$l(x) \quad$ Offset of micro-deformable manipulator in the inertial coordinate system, $\mathrm{m}$

$F \quad$ End force in the free-body diagram, $\mathrm{N}$

$T \quad$ Moment obtained by force analysis, $\mathrm{N} \cdot \mathrm{m}$

$\sigma_{\text {ave }} \quad$ Mean normal stress, $\mathrm{N} / \mathrm{m}^{2}$

$\tau_{s} \quad$ Shear stress, $\mathrm{N} / \mathrm{m}^{2}$

A Cross section area of micro-deformable manipulator, $\mathrm{m}^{2}$

$p_{1} \quad$ Boundary interference of one side

$p_{2} \quad$ Boundary interference of another side

$\hat{p}_{1} \quad$ The estimated value of $p_{1}$

$\hat{p}_{2} \quad$ The estimated value of $p_{2}$

$k_{p} \quad$ The coefficient of control law

$k_{d} \quad$ The coefficient of control law

$k \quad$ The coefficient of control law

$\lambda \quad$ Flexibility of micro-deformable manipulator 


\section{Appendix A}

Design explanation of $k_{p}, k_{d}$, and $k$.

1. According to the engineering trial method, the coefficient $k_{p}$ is taken as 60 .

2. According to Equations (19) $-(21), 0<\alpha<\frac{1}{\left(2 L, 2 \rho L^{3} / E I, 2\left(I_{m}+2 \rho L^{2}\right) / k_{p}, 2\right)}$.

3. From $\eta_{1}=\frac{1}{2} \alpha \rho L, \eta_{2}=E I-\alpha E I L$, we obtain that $\eta_{0}>\max \left(\eta_{1}, \frac{\eta_{1} \eta_{2}}{\eta_{2}-\eta_{1}}\right)$.

4. Take $k_{d}=30$ according to the condition: $\varepsilon_{3}=k_{d}-\alpha I_{m}-k_{d} \alpha>0$; take $k=20$ according to the condition: $\varepsilon_{5}=k-\eta_{0}>0$.

5. Verifying other conditions in Equation (34): $\varepsilon_{1}=\frac{3}{2} \alpha-2 \alpha L^{2}-\frac{2 \alpha L^{3}}{E I}>0, \varepsilon_{2}=\frac{1}{2} \alpha>0$, $\varepsilon_{4}=\alpha k_{p}-k_{d} \alpha-2 \alpha E I L-2 \alpha L^{2}>0, \varepsilon_{5}=k-\eta_{0}>0$.

6. Take $\alpha_{3}=1.5$ according to the condition: $2>\alpha_{3}>1$.

7. From $\min \left(2 \varepsilon_{1}, 2 \varepsilon_{2}, \frac{2 \varepsilon_{3}}{I_{m}}, \frac{2 \varepsilon_{4}}{k_{p}}, \frac{2 \varepsilon_{5}}{m}\right) \geq \lambda_{0}>0$, we obtain $\lambda=\frac{\lambda_{0}}{\alpha_{3}}$.

\section{References}

1. Alandoli, E.A.; Lee, T.S.; Lin, Y.J.; Vijayakumar, V. Dynamic Model and Intelligent Optimal Controller of Flexible Link Manipulator System with Payload Uncertainty. Arab. J. Sci. Eng. 2021. [CrossRef]

2. Jing, Z.L.; Xu, Q.M.; Huang, J.Z. A review on kinematic analysis and dynamic stable control of space flexible manipulators. Aerosp. Syst. 2019, 2, 1-14. [CrossRef]

3. Kharabian, B.; Mirinejad, H. Hybrid sliding mode/H-infinity control approach for uncertain flexible manipulators. IEEE Access 2020, 8, 170452-170460. [CrossRef]

4. Lei, R.H.; Chen, L. Adaptative fault-tolerant and vibration-suppressed hybrid control for flexible space manipulator. J. Astronaut. 2020, 41, 472-482.

5. Zhang, L.J.; Liu, J.K. Adaptive boundary control for flexible two-link manipulator based on partial differential equation dynamic model. IET Control Theory Appl. 2013, 7, 43-51. [CrossRef]

6. He, W.; Ge, S.S.; How, B.V.E.; Choo, Y.S.; Hong, K.-S. Robust adaptive boundary control of a flexible marine riser with vessel dynamics. Automatica 2011, 47, 722-732. [CrossRef]

7. He, W.; He, X.; Zou, M.; Li, H. PDE model-based boundary control design for a flexible robotic manipulator with input backlash. IEEE Trans. Control Syst. Technol. 2019, 27, 790-797. [CrossRef]

8. Gao, H.; He, W.; Zhou, C.; Sun, C. Neural network control of a two-link flexible robotic manipulator using assumed mode method. IEEE Trans. Ind. Inform. 2019, 15, 755-765. [CrossRef]

9. Li, L.; Liu, J.K. Neural-network-based adaptive fault-tolerant vibration control of single-link flexible manipulator. Trans. Inst. Meas. Control 2020, 42, 430-438. [CrossRef]

10. Yang, H.J.; Liu, J.K. An adaptive RBF neural network control method for a class of nonlinear systems. IEEE-CAA J. Autom. Sin. 2018, 5, 457-462. [CrossRef]

11. Zhang, S.; Dong, Y.; Ouyang, Y.; Yin, Z.; Peng, K. Adaptive Neural Control for Robotic Manipulators with Output Constraints and Uncertainties. IEEE Trans. Neural Netw. Learn. Syst. 2018, 29, 5554-5564. [CrossRef] [PubMed]

12. He, C.; Zhang, F.; Jiang, J.H. Adaptive Boundary Control of Flexible Manipulators with Parameter Uncertainty Based on RBF Neural Network. Shock Vib. 2020, 2020. [CrossRef]

13. Ren, Y.; Zhao, Z.; Zhang, C.; Yang, Q.; Hong, K.-S. Adaptive Neural-Network Boundary Control for a Flexible Manipulator with Input Constraints and Model Uncertainties. IEEE Trans. Cybern. 2020. [CrossRef]

14. Zhang, Y.Y.; Kim, D.G.; Zhao, Y.D.; Lee, J.M. PD Control of a Manipulator with Gravity and Inertia Compensation Using an RBF Neural Network. Int. J. Control Autom. Syst. 2020, 18, 3083-3092. [CrossRef]

15. Gu, Y.K.; Liu, H. Adaptative control of flexible-joint manipulator based on dynamics surface and neural networks. J. Huazhong Univ. Sci. Tech. (Nat. Sci. Ed.) 2018, 46, 64-69.

16. Yang, Y.; Liu, Z.; Ma, G. Adaptive distributed control of a flexible manipulator using an iterative learning scheme. IEEE Access 2019, 7, 145934-145943. [CrossRef]

17. Zhou, X.Y.; Wang, H.P.; Tian, Y.; Zheng, G. Disturbance observer-based adaptive boundary iterative learning control for a rigid-flexible manipulator with input backlash and endpoint constraint. Int. J. Adapt. Control Signal Process. 2020, 34, 1220-1241. [CrossRef]

18. Wang, H.; Fu, B.C.; Xue, B.; Yang, C.L. Dynamics analysis of 6-DOF manipulators with flexible joints. China Mech. Eng. 2016, 27, 1096-1101.

19. Wei, Y.K.; Jiao, G.T. Flexible robot arm dynamics analysis based on MATLAB. Mech. Eng. Autom. 2016, 1, 33-36.

20. Liu, X.L.; Huang, Y.P.; Cui, P.J.; Xu, Z.X. Modeling and dynamic characteristic analysis of flexible robotic arm. Noise Vib. Control 2014, 34, 7-11.

21. Endo, T.; Sasaki, M.; Matsuno, F. Contact-force control of a flexible Timoshenko arm. IEEE Trans. Autom. Control 2017, 62, 1004-1009. [CrossRef] 
22. Han, F.; Jia, Y. Boundary control and exponential stability of a flexible Timoshenko beam manipulator with measurement delays. IET Control Theory Appl. 2020, 14, 499-510. [CrossRef]

23. Meng, Q.X.; Lai, X.Z.; Yan, Z.; Wang, Y.W.; Wu, M. Position control without residual vibration for a two-link rigid-flexible manipulator. Control Theory Appl. 2020, 37, 620-628.

24. Sun, L.; Yin, W.; Wang, M.; Liu, J. Position Control for Flexible Joint Robot Based on Online Gravity Compensation with Vibration Suppression. IEEE Trans. Ind. Electron. 2018, 65, 4840-4848. [CrossRef]

25. Pradhan, S.K.; Subudhi, B. Position control of a flexible manipulator using a new nonlinear self-tuning PID controller. IEEE/CAA J. Autom. Sin. 2020, 7, 136-149. [CrossRef]

26. Sun, C.; Gao, H.; He, W.; Yu, Y. Fuzzy neural network control of a flexible robotic manipulator using assumed mode method. IEEE Trans. Neural Netw. Learn. Syst. 2018, 29, 5214-5227. [CrossRef]

27. Dong, F.; Zhao, X.; Han, J.; Chen, Y. Optimal fuzzy adaptive control for uncertain flexible joint manipulator based on D-operation. IET Control Theory Appl. 2018, 12, 1286-1298. [CrossRef]

28. Zhang, C.L.; Yang, T.; Sun, N.; Fang, Y.C. An Adaptive Fuzzy Control Method of Single-Link Flexible Manipulators with Input Dead-Zones. Int. J. Fuzzy Syst. 2020, 22, 2521-2533. [CrossRef]

29. Meirovitch, L. Fundamentals of Vibrations; McGrow Hill: New York, NY, USA, 2002.

30. Rahn, C.D. Mechatronic Control of Distributed Noise and Vibration; Springer: New York, NY, USA, 2001. 